\title{
Unfair Allocation of Gains under the Equal Price Allocation Method in Purchasing Groups
}

\author{
Schotanus, Fredo; Telgen, Jan; Boer, de, Luitzen \\ European Journal of Operational Research xxx (2007) xxx-xxx
}

\begin{abstract}
Certain purchasing groups do not flourish. A supposed reason for this is a creeping dissatisfaction among various members of a group with the allocation of the cooperative gains. In this paper, we analyze unfairness resulting from using the commonly used Equal Price (EP) method for allocating gains under the assumption of continuous quantity discounts. We demonstrate that this unfairness is caused by neglecting a particular component of the added value of individual group members. Next, we develop two fairness ratios and tie these to fairness properties from cooperative game theory. The ratios can be used to assess if EP is an unfair method in specific situations and they show among other things that being too-big a player in a purchasing group can lead to decreasing gains. Finally, we discuss measures a purchasing group could consider in order to attenuate perceived unfairness. Thereby, the group may improve its stability and prosperity.
\end{abstract}

Keywords: Purchasing; Game theory; Group decisions and negotiations; Allocation; Group purchasing

\section{Introduction}

Cooperative purchasing initiatives, such as purchasing groups, purchasing consortia, and buying offices are becoming more and more well-established in the public sector and are gaining popularity in the private sector as well (Carter et al., 2000; Doucette, 1997; Hendrick, 1997; Johnson, 1999; Rozemeijer, 2000; Zentes and Swoboda, 2000). Reasons indicated for this trend are the development of e-procurement (Huber et al., 2004), shifting agendas from a short-term view and internal focus to a long-term view and external relationship focus (Dobler, 1996; Essig, 2000), an increased level of competition and cost pressure (Hendrick, 1997; Johnson, 1999; Nollet and Beaulieu, 2005), an increased awareness and importance of purchasing (Ellram and Carr, 1994), and the wish to counterbalance the power of large suppliers (Nollet and Beaulieu, 2005).

We define cooperative purchasing as the sharing and/or bundling of purchasing related information, processes, resources, and/or volumes by two or more organizations in a purchasing group in order to improve their performances. A purchasing group consists of two or more dependent or independent organizations that purchase together, either formally or informally, or through a third party (Hendrick, 1997). Typical advantages of cooperative purchasing follow from factors like economies of scale (Rozemeijer, 2000), reduction of transaction costs (Johnson, 1999), cost avoidance, and improved relationships with suppliers and other organizations in a purchasing group (Hendrick, 1997). Examples of tangible advantages of cooperative purchasing are reduced purchasing prices (Nollet and Beaulieu, 2003) and reduced workloads (Schotanus, 2005). Reported disadvantages of cooperative purchasing follow from factors such as increased complexity of the purchasing process (Tella and Virolainen, 2005) and loss of flexibility and control (Schotanus, 2005). Examples of tangible disadvantages of cooperative purchasing are increased coordination costs (Johnson, 1999), having to change specifications, and losing existing relations with suppliers

(Schotanus, 2005). 
Despite its increasing popularity in practice, cooperative purchasing has received relatively little attention in management research (Essig, 2000; Tella and Virolainen, 2005). In addition, cooperative purchasing research has focused primarily on inductive explanations of practice and qualitative deductive reasoning (Heijboer, 2003). One specific issue receiving particularly little research attention is the allocation of financial gains resulting from purchasing price savings obtained by cooperative purchasing while using the so-called Equal Price (EP) allocation method. This commonly used EP method is defined as all organizations paying an equal price per item (Heijboer, 2003). It is lamentable that the EP method is not well-studied in literature as financial gains are often an important reason for individual organizations to join a purchasing group (Nollet and Beaulieu, 2003). Therefore, this paper focuses specifically on the EP method.

While practically and intuitively appealing, EP may lead to unfair outcomes under certain circumstances. This has been reported previously by Heijboer (2003), but a systematic analysis of this problem is lacking. Still, reasons reported for failure or stagnation of cooperative purchasing, such as disagreements caused by large differences in organization size (Schotanus, 2004), lack of commitment (Doucette, 1997), anti-trust issues, and fear of free riding organizations (Hendrick, 1997) are often related to the way the purchasing group's gains are distributed (Heijboer, 2003). To prevent these kinds of distribution problems, each of the organizations in a purchasing group should therefore receive a fair part of the total gains (Dyer, 2000). However, this may be difficult when organizations purchase different volumes through a purchasing group and use EP for allocating gains.

An example of one of the problems of EP is illustrated by the so-called hitchhikers' problem (Schotanus, 2005). This problem occurs when a small buying organization uses a contract negotiated by a large buying organization. For large organizations, there may be no incentive to allow hitchhiking while using EP. For small organizations, it can be very interesting to hitchhike though, as they lack economies of scale and can obtain a substantially lower purchasing price by hitchhiking. Granot and Sošic (2005) discuss a similar problem in which a relatively small organization would benefit from joining a specific purchasing group, but the inclusion of such an organization could possibly decrease the profits of the bigger organizations in this exchange. Furthermore, Essig (2000) notes that it is important to avoid an imbalance of incentives and contributions of organizations in a purchasing group, which can be caused by EP. Finally, reasoning from an equity theory perspective (Adams, 1963; Adams, 1965), it can be explained how perceptions of equity are developed. Equity theory states that individuals who feel under-rewarded will try to restore equity. Similarly to purchasing groups, EP may lead to under-rewarded organizations in a group. This may lead to lower commitment of these organizations or them leaving the group (Das, 2001).

Despite the relevance of the studies mentioned above, these studies do not formally analyze how and under which conditions unfairness arises while using EP. These two issues are important to all types of purchasing groups as all of them have to make a decision on how to allocate its gains. Therefore, this paper provides an analytical analysis of unfair outcomes of EP, provides recommendations for purchasing groups as how to deal with it, and contributes to more awareness and understanding of EP related problems. Hence, the main research question in this paper is: how and under which conditions does the Equal Price allocation method lead to unfair outcomes?

The organization of the paper is as follows. First, we develop a formal model of cooperative purchasing that enables us to analyze and illustrate unfairness effects while using EP. Next, we use the formal model of cooperative purchasing to analytically investigate what makes EP result in unfair outcomes. We do this by decomposing the added value of a purchasing group into three components and study how applying EP affects each component separately. This will answer the first part of our research question: how does EP lead to unfair 
outcomes? Next, we study how the degree of unfairness is affected by the relative stake of each organization in a purchasing group and develop several practical guidelines. This will answer the second part of our research question: under which conditions does EP lead to unfair outcomes? In the final sections, we discuss the limitations of the research, draw conclusions, and provide recommendations for purchasing groups and scholars in the field.

\section{A cooperative purchasing model}

As mentioned in the introduction, several issues play a role in the success of establishing and managing purchasing groups. In this paper, we focus on the actual financial gain issue, as this is indicated in the previous section as an important reason for purchasing cooperatively. Hence, we do not consider situations where quantity discounts are dependent on individual transportation costs, decreasing the direct financial gains.

We model a purchasing group by assuming purchasing price savings due to economies of scale when buying from suppliers (Heijboer, 2003). In our model, we make the following three basic assumptions about quantity discounts, which hold for many practical situations (Arnold, 1996; Dolan, 1987; Heijboer, 2003; Schotanus, 2006).

Assumption 1. For the purchasing price per item $p(q)$, we assume that a nonincreasing volume discount is given with more items being purchased. In addition, we assume the total purchasing spend $q \cdot p(q)$ to be increasing with the number of items being bought (Heijboer, 2003).

Assumption 2. Based on Assumption 1, we assume that $p(q)=p_{0} \cdot\left(c_{1}+\frac{c_{2}}{q^{\eta}}\right)$ for $q>0$. This function corresponds with almost all kinds of different types of quantity discounts (Schotanus, 2006). The parameter $\eta$ represents the steepness of the price function for $\eta \geq-1$ and $\eta \neq 0$. For $p_{0}>0$ and $c_{1}>0, p_{0} \cdot c_{1}$ represents the minimum (maximum) price of a function with a positive (negative) $\eta$. Furthermore, $p_{0} \cdot c_{2}$ represents the spread of the function. If $\eta$ is negative (positive), then $c_{2}$ is negative (positive) as well. Still, price functions with a negative $\eta$ are somewhat peculiar because extrapolating such functions leads eventually to negative purchasing prices. Nevertheless, for quite limited ranges, discount schedules with a negative $\eta$ occur in practice (Schotanus, 2006). Such limited ranges may apply to group purchasing situations, but this is usually not the case. Purchasing groups usually purchase relatively large amounts of items.

Assumption 3. For purchasing groups, it may be difficult to determine an accurate estimate for the steepness $\eta$. We assume an average value of 0.50 for a positive $\eta$. This assumption is based on two studies. According to Arnold (1996), doubling the output by concentrating demand as a result of cooperative purchasing can provide on average $25 \%$ reduction of the purchasing price. This corresponds to an average positive $\eta$ of 0.42. According to a study by Schotanus (2006), positive $\eta$ may vary between at least 0.04 and 1.60. In the study, an average positive $\eta$ of 0.58 is found. Therefore, we assume 0.5 to be a reasonable compromise for the average value of $\eta$.

Note that the value of 0.5 for a positive average $\eta$ in Assumption 3 may be questioned. Therefore, we also study the effects of a whole range of values of $\eta$ in Sections 4.2 and 4.3. If 
it is possible to determine an accurate estimate for $\eta$ for the specific purchasing situations of a purchasing group, then a group can consult these results in Sections 4.2 and 4.3.

We refer to our model as a Cooperative Purchasing game or CP game $\left(N, q_{i}, p\right)$ (Heijboer, 2003). $N$ is the total number of organizations in a purchasing group (i.e. the grand coalition), $q$ is the number of items each organization $i$ in a coalition $S$ wants to purchase, and $p$ is the price per item. The total gains function $v(S)$ is defined as the total gains the coalition generates by buying items together compared to the situation where each of the organizations in a purchasing group would buy these items on its own: $v(S)=\sum_{i \in S}\left(q_{i} \cdot p\left(q_{i}\right)\right)-$ $\sum_{i \in S} q_{i} \cdot p\left(\sum_{i \in S} q_{i}\right)$

In the following two sections, we illustrate unfairness by means of a numerical example as a further introduction to our research problem (2.1) and we define the measures of fairness that we shall evaluate in this paper (2.2).

\subsection{Unfairness of Equal Price illustrated}

In the following example, we illustrate the gain allocation effects of current practices in cooperative purchasing. Consider three organizations purchasing 60 items cooperatively and using EP. The price for the items as a function of the quantity that will be ordered is $p(q)=p_{0} \cdot\left(c_{1}+\frac{c_{2}}{q^{0,5}}\right)=959 \cdot\left(1+\frac{1}{\sqrt{q}}\right)$ for $q>0$. This can be modeled into a CP game as is shown in Table 1.

Table 1

CP Game for three organizations

\begin{tabular}{lllll}
\hline $\begin{array}{l}\text { Purchasing } \\
\text { group } S\end{array}$ & $\begin{array}{l}\text { Total quantity } \\
\text { of group } S\end{array}$ & $\begin{array}{l}\text { Price per } \\
\text { item }\end{array}$ & $\begin{array}{l}\text { Total purchasing } \\
\text { volume of group } S\end{array}$ & $\begin{array}{l}\text { Total gains } \\
\text { of group } S\end{array}$ \\
\hline$\{1\}$ & 35 & 1,121 & 39,246 & 0 \\
$\{2\}$ & 10 & 1,262 & 12,625 & 0 \\
$\{3\}$ & 15 & 1,207 & 18,102 & 0 \\
$\{1,2\}$ & 45 & 1,102 & 49,597 & 2,273 \\
$\{1,3\}$ & 50 & 1,095 & 54,741 & 2,607 \\
$\{2,3\}$ & 25 & 1,151 & 28,775 & 1,952 \\
$\{1,2,3\}=N$ & 60 & 1,083 & 64,980 & 4,992 \\
\hline
\end{tabular}

Given this table, the gains can be calculated, which each individual organization receives when the grand purchasing group uses EP:

Organization $i$ gains equalprice $_{i}(v)=q_{i} \cdot\left(p\left(q_{i}\right)-p\left(\sum_{j \in N} q_{j}\right)\right)$

Organization 1 gains $35 \cdot(1,121-1,083)=\mathbf{1 , 3 4 0} \quad$ (largest organization)

Organization 2 gains $10 \cdot(1,262-1,083)=\mathbf{1 , 7 9 5}$ (smallest organization)

Organization 3 gains $15 \cdot(1,207-1,083)=\mathbf{1 , 8 5 7}$

The total gains are $1,340+1,795+1,857=\mathbf{4 , 9 9 2}$

The outcome of this example shows that EP may lead to a situation where the largest organization receives the smallest part of the total gains. The largest organization could object to this allocation as it adds the most value to the purchasing group in our model. This 
situation could lead to instability in the group, because the largest organization could leave or lower its commitment.

\subsection{Properties of fairness for Equal Price}

The unfairness illustrated in the example in Section 2.1 concerns one specific situation. To assess the unfairness of EP in general, we analyze several standard properties of fairness from cooperative game theory (Friedman, 2003; Heijboer, 2003; Moulin, 2001; Shapley, 1953):

(1) EFF: Efficiency. All gains are allocated back to the organizations in a group:

$\sum_{i \in N} f_{i}(v)=v(N)$. Here, $f(v)$ is the allocation vector for each game.

(2) STA: Stability. For all coalitions $S$, it holds that $\sum_{i \in S} f_{i}(v) \geq v(S)$. It means that for each organization the pay-off of cooperation in the group is equal or higher than the pay-off of working alone or in any other subcoalition.

(3) SYM: Symmetry. If two organizations $i$ and $j$ in a group can be interchanged without changing any $v(S)$, then $f_{i}(v)=f_{j}(v)$. It means that equal organizations in a group should get equal pay-offs.

(4) DUM: Dummy. If $v(S \cup\{i\})-v(S)=v(\{i\})$ for all $S \subset N \backslash\{i\}$, then $f_{i}(v)=v(\{i\})$. It means that an organization in a group, who does not contribute anything, should not receive anything.

(5) MON: Monotonicity. If for one organization $i q_{i}^{\prime} \geq q_{i}$, then $f_{i}\left(v^{\prime}\right) \geq f_{i}(v)$. Satisfying this property means that if the quantity of items to be purchased by one organization in a purchasing group stays equal or becomes larger than in a former situation, then this organization should receive an equal or larger amount of gains.

In addition, we introduce one new property of fairness in this paper:

(6) FRAV: Fair Ranking Added Value. If for two organizations $i$ and $j$ in a group $M_{i}(v) \geq M_{j}(v)$, then $f_{i}(v) \geq f_{j}(v)$. Here, $M_{i}(v)=v(N)-v(N \backslash\{i\})$ (Borm et al., 1992), as we will discuss in Section 3.1. Satisfying the FRAV property means that an organization with an equal or larger added value should receive an equal or larger share of the gains.

Table 2 gives an overview of which properties of fairness are satisfied in general for CP games for EP and two other allocation methods, which have been well-studied in the cooperative game theory literature. The table shows that the allocations of the three methods have the core property, i.e. in general, the allocation methods satisfy EFF and STA. In addition, the table shows that FRAV and MON do not depend on EFF, SYM, DUM, and STA for CP games.

The table also shows that EP can lead to situations where an organization adds more value to a group than another organization, but receives fewer gains (i.e. the FRAV property is not satisfied). In addition, the situation could occur in which an organization increases its purchases through the group, but in return receives a smaller amount of the gains (i.e. the MON property is not satisfied). This could slow down further growth of the purchasing group or harm its stability. 
Table 2

Properties of Fairness for CP games

\begin{tabular}{lccc}
\hline Properties of fairness & Equal Price & $\begin{array}{c}\text { Shapley Value } \\
\text { (Shapley, 1953) }\end{array}$ & $\begin{array}{c}\text { Compromise Value } \\
\text { (Borm et al., 1992) }\end{array}$ \\
\hline Efficiency (EFF) & $\checkmark$ & $\checkmark$ & $\checkmark$ \\
Stability (STA) & $\checkmark$ & $\checkmark$ & $\checkmark$ \\
Symmetry (SYM) & $\checkmark$ & $\checkmark$ & $\checkmark$ \\
Dummy (DUM) & $\checkmark$ & $\checkmark$ & $\checkmark$ \\
Monotonicity (MON) & $\boldsymbol{x}$ & $\checkmark$ & $\checkmark$ \\
Fair Ranking Added Value (FRAV) & $\mathbf{x}$ & $\mathbf{x}$ & $\checkmark$ \\
\hline
\end{tabular}

Note: $\checkmark=$ satisfied in general, $\mathbf{x}=$ not satisfied in general

Although useful, Table 2 only gives a general overview of fairness properties. The table does not provide information for purchasing groups on how and under which specific conditions EP leads to unfair outcomes.

\section{How does Equal Price lead to unfair outcomes?}

In this section, we extend the model developed in the previous section in order to investigate the underlying mechanism that causes EP to produce unfair outcomes. This will answer the first part of our research question: how does EP lead to unfair outcomes? We answer this question by formally defining the added value of an organization to a purchasing group in Section 3.1, breaking it down into three components in Section 3.2, and proving that EP neglects one component of the added value in Section 3.3.

\subsection{Added value}

Organizations can add value to a group in several ways. In this paper, the added value of each organization $i$ for the other organizations of a group is defined as the total gains of the group minus the gains the other organizations in the group can establish without organization $i: M_{i}(v)=v(N)-v(N \backslash\{i\})$. Given $M_{i}(v)$, we can calculate the added value of the organizations 1, 2, and 3 from Section 2.1:

The added value of organization $i$ is $M_{i}(v)=v(\{1,2,3\})-v(\{1,2,3\} \backslash\{i\})$

The added value of organization 1 is 4,992-1,952 = 3,040 (largest organization)

The added value of organization 2 is $4,992-2,607=2,385 \quad$ (smallest organization)

The added value of organization 3 is $4,992-2,273=\mathbf{2 , 7 1 9}$

\subsection{Decomposing added value}

To obtain more insight into the value that organizations add to a group, we split the added value of an organization into three different components as we also show in Theorem 1: (1) gains for and by organization $i$ created by joining a group $\left(m_{i}\right),(2)$ gains created by organization $i$ for the other organizations in a group $\left(n_{i}\right)$, and (3) gains for organization $i$ created by the other organizations in a group $\left(o_{i}\right)$. For instance, the added value of the organizations from Section 2.1 can be divided into these three types of gains as is shown in Table 3. Fig. 1 and 2 illustrate how the three components work together in creating and limiting value.

In Fig. 1, a price per item of 1,207 applies to a quantity of 15 (quantity of organization 3). A price of 1,102 applies to a quantity of 45 (quantity of the other organizations) and a price of 1,083 applies to a total quantity of 60. In Fig. 2, a price of 1,121 applies to a quantity of 35 (quantity of organization 1), a price of 1,141 applies to a quantity of 25 (quantity of the other 
organizations) and a price of 1,083 applies to a total quantity of 60 . The corresponding values of $n_{i}, m_{i}$, and $o_{i}$ are shown in Table 3 . Note that $o_{1}$ is 0 as organization 1 purchases more than half of the total purchasing volume of the purchasing group.

Table 3

Decomposition of added value into three types of gains

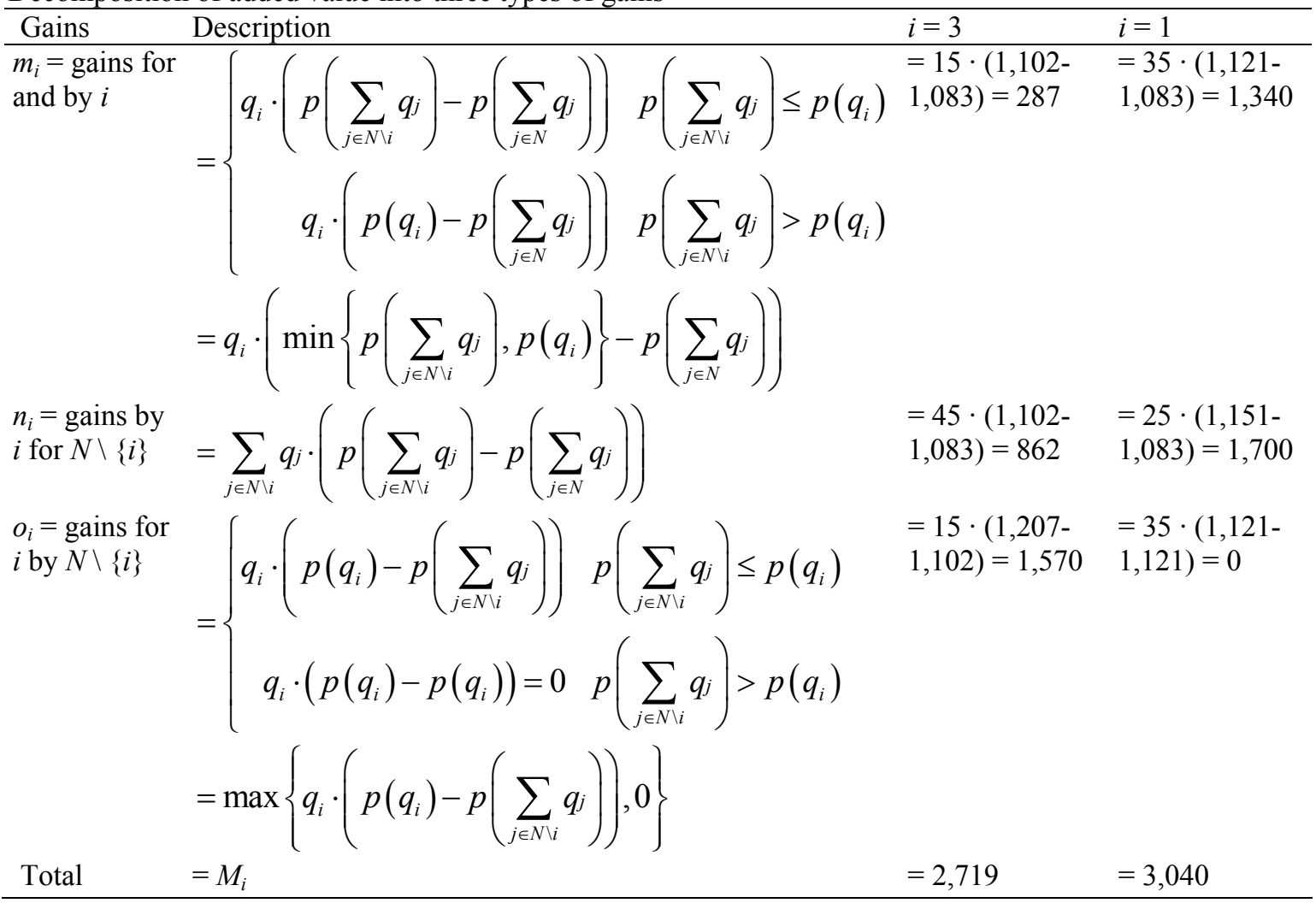

Fig. 1. Decomposition of added value into three types of gains while $p\left(\sum_{j \in N \backslash i} q_{j}\right) \leq p\left(q_{i}\right)$ and $i=3$

Fig. 2. Decomposition of added value into two types of gains while $p\left(\sum_{j \in N \backslash i} q_{j}\right)>p\left(q_{i}\right)$ and $i=1$

Theorem 1. The added value $M_{i}(v)=v(N)-v(N \backslash\{i\})$ of organization $i$ in a purchasing group can be split in three different components $M_{i}(v)=m_{i}+n_{i}+o_{i}$ as are defined in Table 3.

Proof. If the value of a purchasing group $v(N)=\sum_{j \in N}\left(q_{j} \cdot p\left(q_{j}\right)\right)-\sum_{j \in N} q_{j} \cdot p\left(\sum_{j \in N} q_{j}\right)$, then the added value of an organization $i$ for a purchasing group is $M_{i}(v)=v(N)-v(N \backslash\{i\})$ 
$=\sum_{j \in N}\left(q_{j} \cdot p\left(q_{j}\right)\right)-\sum_{j \in N} q_{j} \cdot p\left(\sum_{j \in N} q_{j}\right)-\sum_{j \in N \backslash i}\left(q_{j} \cdot p\left(q_{j}\right)\right)+\sum_{j \in N \backslash i} q_{j} \cdot p\left(\sum_{j \in N \backslash i} q_{j}\right) \cdot$ We can rewrite this as $M_{i}(v)=q_{i} \cdot p\left(q_{i}\right)-\sum_{j \in N} q_{j} \cdot p\left(\sum_{j \in N} q_{j}\right)+\sum_{j \in N \backslash i} q_{j} \cdot p\left(\sum_{j \in N \backslash i} q_{j}\right)$, as

$M_{i}(v)=q_{i} \cdot\left(p\left(q_{i}\right)-p\left(\sum_{j \in N} q_{j}\right)\right)+\sum_{j \in N \backslash i} q_{j} \cdot\left(p\left(\sum_{j \in N \backslash i} q_{j}\right)-p\left(\sum_{j \in N} q_{j}\right)\right)$, and as

$M_{i}(v)=q_{i} \cdot\left(\min \left\{p\left(\sum_{j \in N \backslash i} q_{j}\right), p\left(q_{i}\right)\right\}-p\left(\sum_{j \in N} q_{j}\right)\right)+\sum_{j \in N \backslash i} q_{j} \cdot\left(p\left(\sum_{j \in N \backslash i} q_{j}\right)-p\left(\sum_{j \in N} q_{j}\right)\right)$

$+\max \left\{q_{i} \cdot\left(p\left(q_{i}\right)-p\left(\sum_{j \in N \backslash i} q_{j}\right)\right), 0\right\}$. So, given the definitions of $\mathrm{m}_{\mathrm{i}}, \mathrm{n}_{\mathrm{i}}$, and $\mathrm{o}_{\mathrm{i}}$ in Table 3 , the added value of an organization $i$ for a purchasing group is $M_{i}(v)=m_{i}+n_{i}+o_{i}$.

\subsection{Equal Price neglects one component of added value}

We illustrated in Sections 2.1 and 2.2 that EP can be unfair in situations where organizations differ in size. Now, given the three components of the added value we can prove that this unfairness is caused by the fact that EP neglects component $n_{i}$ of the added value of an organization for a group, as is shown in Theorem 2:

Theorem 2. The Equal Price concept neglects the component $n_{i}$ of the added value of organization i for a purchasing group, as the added value of organization i for a purchasing group is $M_{i}(v)=m_{i}+n_{i}+o_{i}$ and the Equal Price allocation for organization $i$ is equalprice $_{i}(v)=m_{i}+o_{i}$.

Proof. In Theorem 1, we have already proven that the added value of an organization $i$ for a group is $M_{i}(v)=m_{i}+n_{i}+o_{i}$. So, we only have to prove that equalprice $(v)=m_{i}+o_{i}$. We do this by rewriting the definition of equalprice $(v)=q_{i} \cdot\left(p\left(q_{i}\right)-p\left(\sum_{j \in N} q_{j}\right)\right)$. We can rewrite this as equalprice ${ }_{i}(v)=\left\{\begin{array}{ll}q_{i} \cdot\left(p\left(q_{i}\right)-p\left(\sum_{j \in N} q_{j}\right)\right) & p\left(\sum_{j \in N \backslash i} q_{j}\right) \leq p\left(q_{i}\right) \\ q_{i} \cdot\left(p\left(q_{i}\right)-p\left(\sum_{j \in N} q_{j}\right)\right) & p\left(\sum_{j \in N \backslash i} q_{j}\right)>p\left(q_{i}\right)\end{array}\right.$ and as equalprice $_{i}(v)=\left\{\begin{array}{rr}q_{i} \cdot\left(p\left(q_{i}\right)+p\left(\sum_{j \in N \backslash i} q_{j}\right)-p\left(\sum_{j \in N \backslash i} q_{j}\right)-p\left(\sum_{j \in N} q_{j}\right)\right) & p\left(\sum_{j \in N \backslash i} q_{j}\right) \leq p\left(q_{i}\right) \\ q_{i} \cdot\left(p\left(q_{i}\right)-p\left(\sum_{j \in N} q_{j}\right)\right)+0 & p\left(\sum_{j \in N \backslash i} q_{j}\right)>p\left(q_{i}\right)\end{array}\right.$.

Now, it follows from Table 3 that equalprice $(v)=$ 
$q_{i} \cdot\left(\min \left\{p\left(\sum_{j \in N \backslash i} q_{j}\right), p\left(q_{i}\right)\right\}-p\left(\sum_{j \in N} q_{j}\right)\right)+\max \left\{q_{i} \cdot\left(p\left(q_{i}\right)-p\left(\sum_{j \in N \backslash i} q_{j}\right)\right), 0\right\}$. So, the

Equal Price allocation for organization $i$ is equalprice $(v)=m_{i}+o_{i}$.

We have shown in our example in Section 2.1 that the largest organization may receive the smallest part of the total gains despite adding the most value. Using Theorem 2, we demonstrate that this is caused by ignoring $n_{i}$ :

Organization $i$ gains equalprice $e_{i}(v)=m_{i}+o_{i}$

Organization 1 gains $m_{1}+o_{1}=\mathbf{1 , 3 4 0} \quad$ (largest organization)

Organization 2 gains $m_{2}+o_{2}=\mathbf{1 , 7 9 5} \quad$ (smallest organization)

Organization 3 gains $m_{3}+o_{3}=\mathbf{1 , 8 5 7}$

The added value of organization $i$ is $M_{i}(v)=m_{i}+n_{i}+o_{i}$

The added value of organization 1 is $\left(m_{1}+o_{1}\right)+n_{l}=1,340+1700=\mathbf{3 , 0 4 0}$

The added value of organization 2 is $\left(m_{2}+o_{2}\right)+n_{2}=1,795+591=\mathbf{2 , 3 8 5}$

The added value of organization 3 is $\left(m_{3}+o_{3}\right)+n_{3}=1,857+862=\mathbf{2 , 7 1 9}$

\section{Under which conditions does Equal Price lead to unfair outcomes?}

In this section, we investigate which circumstances determine the extent of unfairness caused by EP. In Section 4.1, we analyze the three components of the added value of organizations for a group. Proofs have been omitted in Section 4.1; instead we combine the effects on the three components in two theorems which come with proofs in Sections 4.2 and 4.3. In these two sections, we also show how we can use our results to develop practical guidelines that purchasing groups can use to enhance their stability and prosperity. This will answer the second part of our research question: under which conditions does EP lead to unfair outcomes?

\subsection{The impact of organization size on $m_{i}, n_{i}$, and $o_{i}$}

In our model, there are two main disadvantages to EP, which apply especially to large organizations in a purchasing group. First, as $n_{i}$ is always increasing with more items being purchased by organization $i$, it becomes less attractive for larger organizations to use EP. After all, $n_{i}$ is not incorporated in EP, and the larger the value of $n_{i}$, the more these organizations are put at a disadvantage. The second disadvantage applies to $m_{i}$ and $o_{i}$. These components of added value will become smaller after a certain point. This also puts larger organizations at a disadvantage, because $m_{i}$ and $o_{i}$ are the only two components incorporated in EP.

\subsection{The MON Fairness Ratio}

Fig. 3 illustrates the combined effects of changes in $q_{2}$ on the three different types of gains for organization 2 while the steepness $\eta$ is 0.5 . The total number of needed items for organizations 1 and 3 is constant in this figure. 


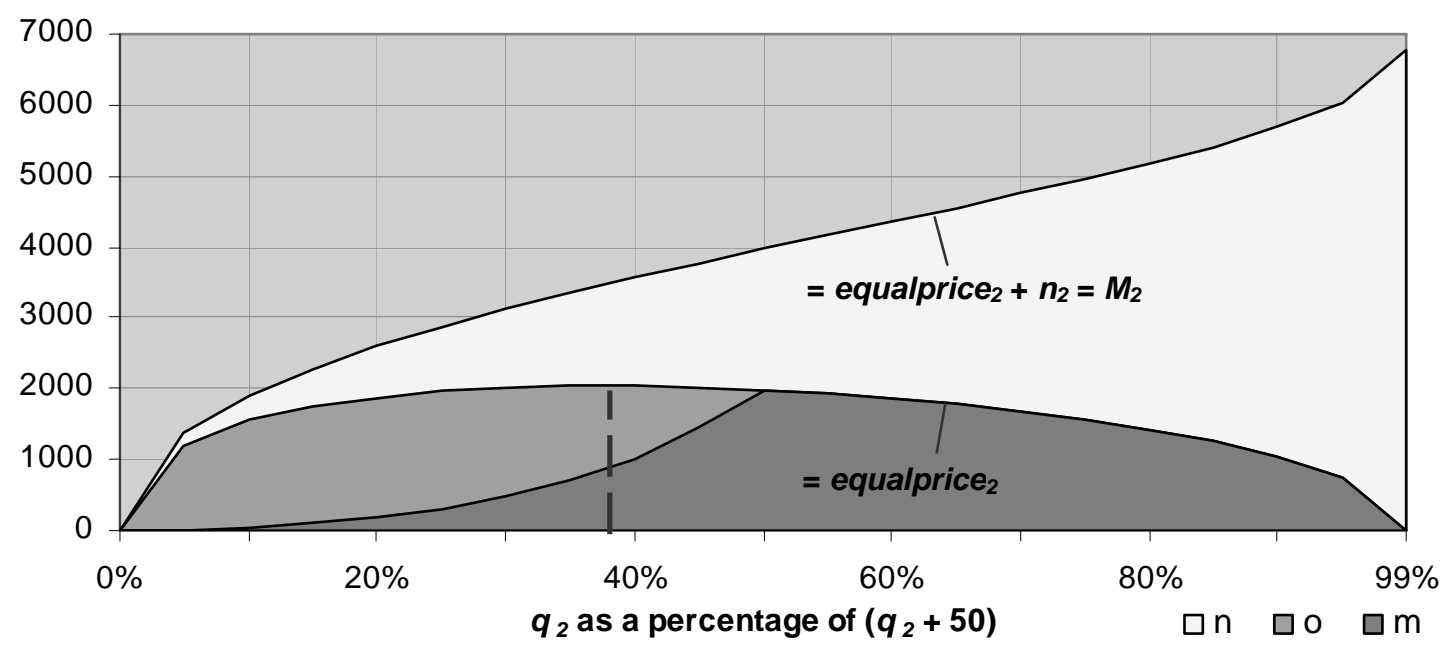

Fig. 3. Type of gains as a function of $q_{2}$ while $q_{1+3}$ is constant and $\eta=0.5$

In this figure, the value of $M_{2}$ increases with an increasing value of $q_{2}$. At the point where $q_{2}$ becomes $38 \%$ of the total volume, the EP outcome for organization 2 reaches its maximum value. We call $38 \%$ the MON Fairness Ratio (MONFR) of EP while $\eta$ is 0.5 . With Theorem 3 , we prove that this is the case in almost any given situation in our model, as this percentage is independent of the values of the parameters $p_{o}, c_{1}$, and $c_{2}$ in the price structure, the number of organizations in a purchasing group, and the allocation of the group volume among these organizations.

Theorem 3. While using the Equal Price concept and given Assumptions 1 to 3, organizations increasing their purchases by a purchasing group to more than $38 \%$ of the total volume are put at a disadvantage; they will receive fewer gains with an increasing volume. Note that this implies that MON is not satisfied from this point.

Proof. Again, the definition of equalprice ${ }_{i}(v)=q_{i} \cdot\left(p\left(q_{i}\right)-p\left(T_{i}\right)\right), \eta=0.5$, and $T_{i}=q_{i}+\sum_{j \in N / i} q_{j}$, where the volume of the other organizations $\sum_{j \in N / i} q_{j}$ is constant. We can rewrite equalprice $(v)$ as $q_{i} \cdot\left(p_{0} \cdot\left(c_{1}+\frac{c_{2}}{\sqrt{q_{i}}}\right)-p_{0} \cdot\left(c_{1}+\frac{c_{2}}{\sqrt{T_{i}}}\right)\right)$. The variable $\mathrm{c}_{1}$ cancels itself out, which gives $q_{i} \cdot\left(p_{0} \cdot\left(\frac{c_{2}}{\sqrt{q_{i}}}\right)-p_{0} \cdot\left(\frac{c_{2}}{\sqrt{T_{i}}}\right)\right)=p_{0} \cdot c_{2} \cdot \sqrt{q_{i}}-\frac{p_{0} \cdot c_{2} \cdot q_{i}}{\sqrt{T_{i}}}$. We want to find the maximum value of equalprice ${ }_{i}(v)$, so, equalprice $e_{i}(v)^{\prime}=$ $\frac{p_{0} \cdot c_{2}}{2 \sqrt{q_{i}}}-\frac{p_{0} \cdot c_{2}}{\sqrt{T_{i}}}+\frac{p_{0} \cdot c_{2} \cdot q_{i}}{2 T_{i}^{1,5}}=\frac{1}{2 \sqrt{q_{i}}}-\frac{1}{\sqrt{T_{i}}}+\frac{q_{i}}{2 T_{i}^{1,5}}=0$. If $q_{i}=\operatorname{MONFR} \cdot T_{i}$, then $\frac{1}{2 \sqrt{M O N F R \cdot T_{i}}}-\frac{1}{\sqrt{T_{i}}}+\frac{M O N F R \cdot T_{i}}{2 T_{i}^{1,5}}=0$. We can rewrite this as $\frac{1}{\sqrt{M O N F R} \cdot \sqrt{T_{i}}}-$ 


$$
\begin{aligned}
& \frac{2}{\sqrt{T_{i}}}+\frac{M O N F R}{\sqrt{T_{i}}}=0, \text { which gives MONFR }+\frac{1}{\sqrt{M O N F R}}-2=0 . \text { So, } \\
& \text { MONFR }=\frac{3-\sqrt{5}}{2} \cdot 100 \%=38 \% .
\end{aligned}
$$

The only dependent variable in this proof is the steepness parameter $\eta$ in the price function $p\left(q_{i}\right)=p_{0} \cdot\left(c_{1}+\frac{c_{2}}{q_{i}{ }^{\eta}}\right)$ for $q_{i}>0$. Until now, we assumed $\eta$ always being 0.5 .

However, 0.5 is an estimated average value as discussed in Assumption 3. In practice, $\eta$ may vary. For values of $\eta$ between -1 and 1 , the following applies:

$\eta \cdot \mathrm{MONFR}^{1+\eta}-\mathrm{MONFR}^{\eta}-\eta+1=0$ (see also Fig. 4). Note that $\eta$ less than 0 implies that $c_{2}$ becomes less than 0 and $p_{0} \cdot c_{1}$ becomes the maximum price. For instance, if $\eta$ is $-1, p_{0}$ is $959, c_{1}$ is 1 , and $c_{2}$ is -1 , then the price function is $p\left(q_{i}\right)=959 \cdot\left(1-q_{i}\right)$.

If $\eta$ is another value than the estimated average value of 0.5 , then MONFR is not equal to $38 \%$. For instance, if $\eta$ is 1 , then MONFR is $0 \%$ and all organizations increasing their volume through the purchasing group will receive fewer gains. In this case, the smallest organization will receive the largest part of the gains. The largest organization will receive the smallest part of the gains. So, MON is not satisfied in any situation for purchasing groups with various members. With $\eta$ less than $0, \mathrm{MON}$ is satisfied in all situations.

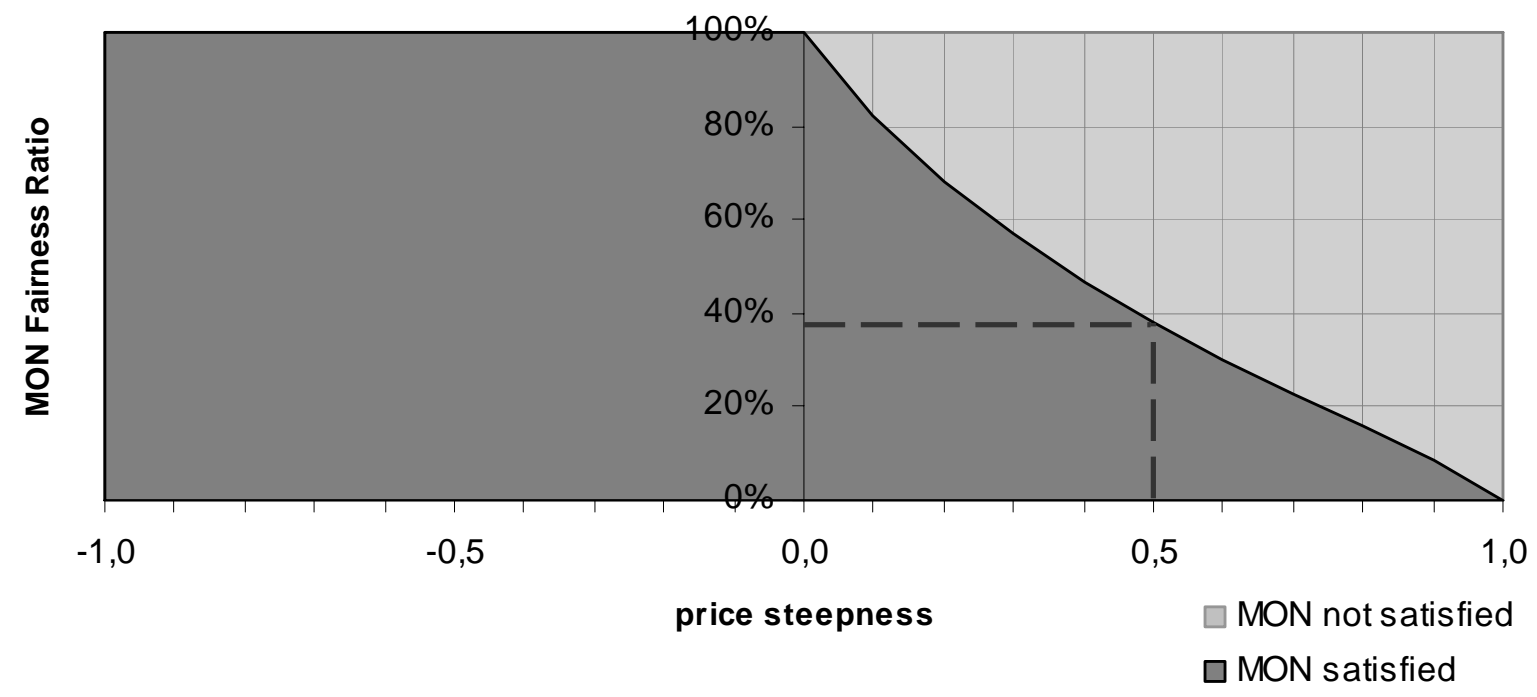

Fig. 4. The MON Fairness Ratio as a function of $\eta$

The main application of Fig. 3 and 4 is that they can help in decisions concerning whether or not to use EP in purchasing groups. If organizations in a purchasing group are unequal in size or size differences among previously similar organizations increase steadily, then it can be easily shown whether or not MON is satisfied and whether or not EP is theoretically fair.

Fig. 3 and 4 can also be applied in situations as in the following example. Consider several organizations in a purchasing group using EP and purchasing different items cooperatively. One organization in this group has the possibility to increase its purchasing volume for one item. This organization can choose between items A and B, which both have an almost identical price function with $\eta$ is 0.5 . For item $A$, the organization can increase its purchasing volume from $30 \%$ to $35 \%$. For item B, the organization can increase its purchasing volume from $40 \%$ to $60 \%$. If this organization wants to optimize its own gains by 
purchasing through the purchasing group, the organization should choose item A. If this organization wants to optimize the total gains of the purchasing group, the organization should choose item B.

To conclude, when using EP and given Assumptions 1 to 3, organizations that increase their stake in the cooperative volume past the $38 \%$ point will receive fewer gains, even though the added value of the organization and the total gains of a purchasing group increase. The more the organizations of a purchasing group will differ in purchasing volumes, the stronger the unfair effects of EP will be. The unfair effects will also be stronger if $\eta$ becomes larger than the assumed average value of 0.5 .

\subsection{The FRAV Fairness Ratio}

Fig. 3 and 4 and the MON property of fairness apply to organizations increasing or decreasing their volume and simultaneously increasing or decreasing the total volume of a purchasing group. Thus, Fig. 3 and 4 and the MON property apply to a dynamic situation. In this section, we study the FRAV property of fairness. This property applies to a stable situation; given a certain allocation of the total volume of a group among individual organizations, we provide guidelines to test whether or not FRAV is satisfied. Therefore, we consider the situation where the total volume of a group is constant, but the allocations of the total volume among the individual organizations differ. In other words, we study the EP effects on all possible allocations that may occur in a purchasing group. Fig. 5 illustrates this scenario for different quantities of organization 2 while the steepness $\eta$ is 0.5 . Figures of the same kind can be drawn for different values of the total volume.

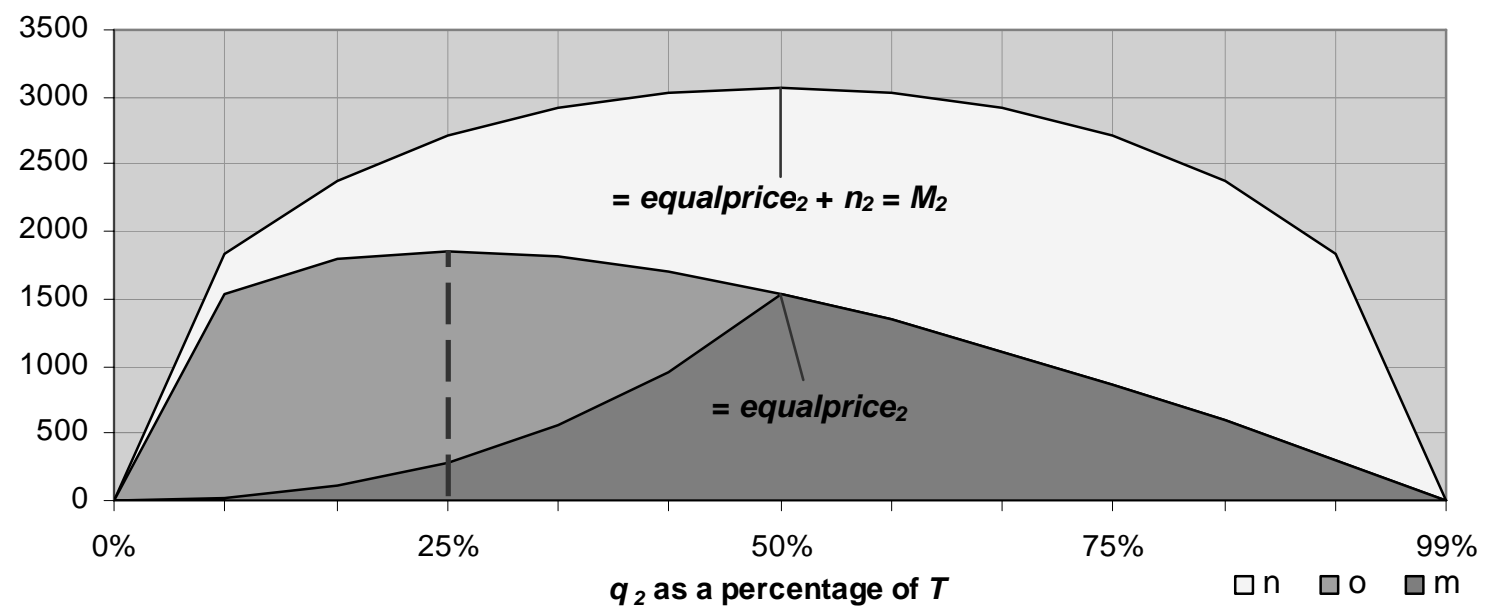

Fig. 5. Type of gains as a function of $q_{2}$ while $T$ is constant and $\eta=0.5$

At the point where $q_{2}$ is $50 \%$ of the total volume, $M_{2}$ reaches its maximum value. However, already at the point where $q_{2}$ is $25 \%$ of the total volume, the EP outcome for organization 2 reaches its maximum. In other words, if $\eta$ is 0.5 for a purchasing group, then the figure shows that an organization that purchases 15 items $(25 \%)$ of a total volume of 60 receives the most gains. Other organizations that purchase $35(58 \%)$ and 10 items $(17 \%)$ receive less gains.

We call 25\% the FRAV Fairness Ratio (FRAVFR) of EP while $\eta$ is 0.5 . With Theorem 4, we prove that this is almost always the case in any given situation in our model. Again, this percentage is independent of the values of the parameters $p_{o}, c_{1}$, and $c_{2}$ in the price structure, the number of organizations in a purchasing group, and the allocation of the group volume among these organizations. 
Theorem 4. While using the Equal Price concept and given Assumptions 1 to 3, organizations purchasing $25 \%$ of the total volume of a purchasing group receive the maximum allocation of gains. Note that this implies that FRAV is not satisfied from this point.

Proof. Again, the definition of equalprice $(v)=q_{i} \cdot\left(\operatorname{price}\left(q_{i}\right)-\operatorname{price}(T)\right), \eta=0.5$, and $T=\sum_{j \in N} q_{j}$ where the total volume of all organizations $\mathrm{T}$ is constant. We can rewrite equalprice $_{i}(v)$ as equalprice $(v)=p_{0} \cdot c_{2} \cdot \sqrt{q_{i}}-\frac{p_{0} \cdot c_{2} \cdot q_{i}}{\sqrt{T}}$. Here, equalprice $_{i}(v)^{\prime}=\frac{p_{0} \cdot c_{2}}{2 \sqrt{q_{i}}}-\frac{p_{0} \cdot c_{2}}{\sqrt{T}}$. Again, we want to find the maximum value of equalprice $_{i}(v)$, so $\frac{p_{0} \cdot c_{2}}{2 \sqrt{q_{i}}}-\frac{p_{0} \cdot c_{2}}{\sqrt{T}}=\frac{1}{2 \sqrt{q_{i}}}-\frac{1}{\sqrt{T}}=0$, which gives $q_{i}=\frac{T}{4}$. So, if $q \cdot 100 \%=F R A V F R \cdot T$, then $F R A V F R=25 \%$.

Once more, the only dependent variable in this proof is the steepness parameter $\eta$. Therefore, organizations in a purchasing group should check the average $\eta$ of the items purchased through the group and the related influence on MONFR and FRAVFR.

We have already described the function of MONFR. For FRAVFR, the following optimality condition applies: FRAVFR $=(1-\eta)^{\frac{1}{\eta}} \leq$ MONFR (see also Fig. 6). For instance, if $\eta$ is -1 , then FRAVFR is $50 \%$. This is a fair situation, because FRAVFR equals the point where the added value also reaches its maximum. If $\eta$ is greater than -1 , then FRAVFR is less than $50 \%$. This could lead to an unfair situation, because FRAVFR reaches its maximum before the added value does.

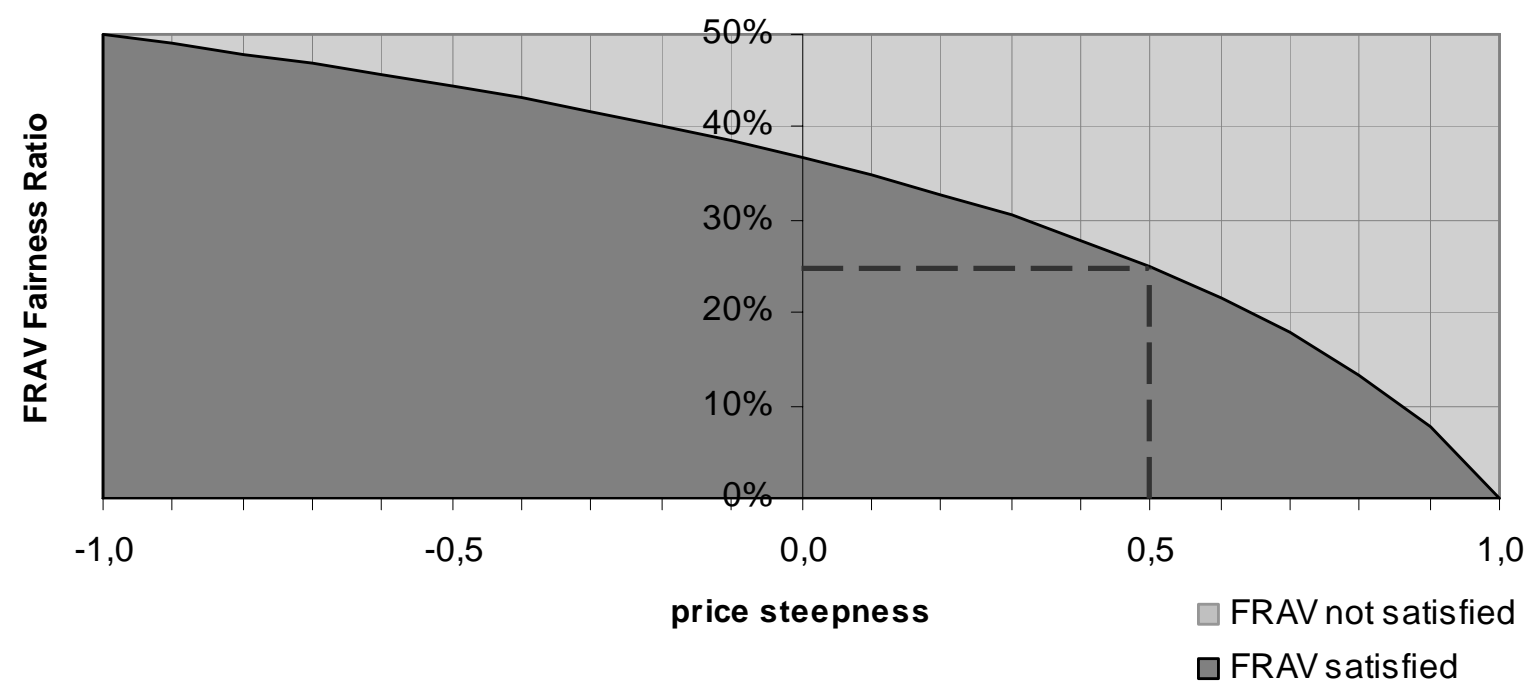

Fig. 6. The FRAV Fairness Ratio as a function of $\eta$

The main application of Fig. 5 and 6 is that they can also serve in decisions concerning whether or not to use EP in purchasing groups. If organizations in a purchasing group are unequal in size or size differences among previously similar organizations increase steadily, then it can be easily shown whether or not FRAV is satisfied and EP is theoretically fair. For instance, if the average $\eta$ is 0.5 , then EP is unfair for purchasing groups with organizations 
larger than $25 \%$ of the total volume and especially to organizations larger than $38 \%$, as both MON and FRAV are not satisfied from this point.

Fig. 5 and 6 can also be useful for finding ways of limiting unfairness effects when a purchasing group uses EP. Consider for example that organizations in a purchasing group want to purchase one new item cooperatively. One organization has the decisive vote in what item to purchase cooperatively. This organization can choose between items A and B, which both have an identical total volume, an almost identical average price function, and $\eta$ is 0.5 . For item A, the organization would purchase $25 \%$ of the total volume. For item B, the organization would purchase $35 \%$ of the total volume. If this organization wants to optimize its own gains by purchasing through the purchasing group, then the organization should choose item A.

To conclude, when using EP and given Assumptions 1 to 3, organizations purchasing $25 \%$ of the total volume will receive the maximum allocation of gains. Larger and smaller organizations will receive a smaller amount of gains. Again, the unfair effects of EP will be stronger if $\eta$ becomes larger than the assumed average value of 0.5 .

\section{Limitations and further research}

Before we draw conclusions on the basis of our analyses in the previous sections, we point out the main limitations of the research that should be taken into account.

First, using a continuous price function may be questioned. In practice, gradual prices are usually used to establish quantity discounts (Munson and Rosenblatt, 1998). For instance, in a gradual price schedule, a price of 400 could apply to 50-99 items and a price of 390 could apply to 100-199 items. Nonetheless, purchasing prices and lot sizes are mostly determined through negotiations (Munson and Rosenblatt, 1998). So, if an organization needs 98 items, then it will usually negotiate a lower price than 400 or otherwise it will order 100 items. In contrast to continuous price functions, gradual price functions do not incorporate this flexibility. Therefore, we use a continuous price function in stead of a gradual price function.

We also note that several researchers have proposed and applied continuous price functions in their studies (Dada and Srikanth, 1987; Dolan, 1987; Jeuland and Shugan, 1983; Rosenblatt and Lee, 1985; Viswanthan and Wang, 2003). We are aware that some of these authors, like Dada and Srikanth (1987), use more complex price functions. However, all different forms of gradual prices found in practice can reliably be approximated by the relatively simple continuous price function used in this paper (Schotanus, 2006).

Second, purchasing groups often purchase multiple items cooperatively. For instance, organization 1 purchases 10 pieces of item A and 100 pieces of item B. Organization 2 purchases 100 pieces of item $A$ and 10 pieces of item B. This could compensate for unfair effects. Therefore, our model is primarily relevant for purchasing groups with an overall variety of members in terms of size.

Third, we neither take into account the costs of cooperating nor advantages other than financial gains. These other advantages, as political control over other organizations or obtaining knowledge from other organizations, could compensate for unfairness related to EP. Furthermore, in some cases, a smaller organization may be able to negotiate a lower price than a larger organization. Obviously, the suitability of a purchasing group may be questioned here.

Further research could (1) take into account the costs of setting up a purchasing group, handling, and monitoring its transactions, (2) take more benefits of cooperation into account than just volume discounts, for instance by using multi-attribute utility (MAUT) functions (Keeney and Raiffa, 1976), and (3) find solution concepts to unfairness problems that take into account all components of the added value of cooperating organizations. 


\section{Conclusions and recommendations}

In this paper, we analyze causes of unfairness resulting from using the Equal Price (EP) allocation method. We first demonstrate that EP may result in unfair allocations of gains for large organizations in purchasing groups. We prove that EP results in these kinds of unfair outcomes because it ignores an important part of the added value of each organization for the other organizations of a purchasing group. This answers our first research question: how does EP lead to unfair outcomes? In the next subsections, we answer our second research question: under which conditions does EP lead to unfair outcomes?

We conclude that under Assumptions 1 to 3 and while using EP, organizations increasing their volume past $38 \%$ of the total volume of a purchasing group will receive fewer gains, even though their added value for the purchasing group increases and the total gains of the group increase. This means that the MON property of fairness is not satisfied past this point. The $38 \%$ guideline applies to an estimated average steepness $\eta$ of 0.5 . We have generalized the $38 \%$ guideline for all values of $\eta$ in the MON Fairness Ratio in Fig. 4.

Furthermore, we prove that under Assumptions 1 to 3 and while using EP, an organization in a purchasing group receives its maximum pay-off when its share of the total volume of a group is $25 \%$. Past this point, the FRAV property of fairness is not satisfied. As a result, it becomes less attractive for larger organizations to participate in a purchasing group. Again, the $25 \%$ guideline only applies to an $\eta$ of 0.5 . We have generalized the $25 \%$ guideline for all values of $\eta$ in the FRAV Fairness Ratio in Fig. 6. Fig. 3 to 6 show that the unfair effects of EP become stronger if $\eta$ becomes larger than the assumed average value of 0.5 .

To conclude, if organizations in a purchasing group are unequal in size or size differences among previously similar organizations increase steadily and they use EP, then it seems important that they address the possible unfairness of EP and develop solutions for it in order to avoid instability of the group on the longer term. Under Assumptions 1 to 3, this applies to purchasing groups with organizations larger than $25 \%$ of the total volume and especially to purchasing groups with organizations larger than 38\%, because both MON and FRAV are not satisfied from this point. Again, for other values of $\eta$, we refer to Fig. 4 and Fig. 6. Possible solutions to EP related problems are the following:

(1) To create a group structure in which the side effects of EP are reduced to a minimum. The MON Fairness Ratio and the FRAV Fairness Ratio can be used to find the right number of organizations of the right size for the purchasing group;

(2) To use another gain allocation mechanism than EP. Cooperative game theory offers several alternative solution concepts like the Shapley Value (Shapley, 1953), generalizations of the Shapley Value (Hamlen et al., 1980; Loehman and Whinston, 1976), the Compromise Value (Borm et al., 1992) or the Adapted Compromise Value (Schotanus, 2004). Again, the MON Fairness Ratio and the FRAV Fairness Ratio can be used to find out if EP is a fair allocation method for the purchasing group or if another allocation method should be used;

(3) To compensate the unfair effects of EP by a costs allocation mechanism that favors larger organizations in a purchasing group. The costs of a purchasing group could be allocated equally or even disproportionally. Again, the MON Fairness Ratio and the FRAV Fairness Ratio can be used to find out if it is necessary to compensate unfair effects of EP. 


\section{Acknowledgements}

We thank the referees for their thoughtful and thorough review. We also acknowledge partial financial support from the Dutch Association for Purchasing Management (NEVI).

\section{References}

Adams JS. Toward an understanding of equity. Journal of Abnormal and Social Psychology $1963 ; 67 ; 422-436$.

Adams JS. Inequity in social exchange. In: L Berkowitz (Ed), Advances in experimental social psychology. Academic Press: New York; 1965.

Arnold U. Purchasing consortia: Theoretical framework and empirical data. Revista de Economia e Direito 1996; 1; 5-26.

Borm P, Keiding H, Mclean R, Oortwijn S, Tijs S. The compromise value for NTU-games. International Journal of Game Theory $1992 ; 21 ; 175-189$.

Carter PL, Carter JR, Monczka RM, Slaigh TH, Swan AJ. The future of purchasing and supply: a ten-year forecast. Journal of Supply Chain Management 2000; 36; 14-26.

Dada M, Srikanth KN. Pricing policies for quantity discounts. Management Science 1987; $33 ; 1247-1252$.

Das TK, Teng BS. A risk perception model of alliance structuring. Journal of International Management 2001; 7; 1-29.

Dobler DW. Purchasing and supply management: text and cases. McGraw-Hill: New York; 1996.

Dolan RJ. Quantity discounts: managerial issues and research opportunities. Marketing Science $1987 ; 6 ; 1-22$.

Doucette WR. Influences on member commitment to group purchasing organizations. Journal of Business Research 1997; 40; 183-189.

Dyer JH. Collaborative advantage, winning through extended enterprise supplier networks. Oxford University Press: New York; 2000.

Ellram LM, Carr A. Strategic purchasing: a history and review of the literature. International Journal of Purchasing and Materials Management 1994; 30; 10-18.

Essig M. Purchasing consortia as symbiotic relationships: developing the concept of consortium sourcing. European Journal of Purchasing and Supply Management 2000; 6; $13-22$.

Friedman EJ. Strong monotonicity in surplus sharing. Economic Theory 2003; 23; 643-658.

Granot D, Sošic G. Formation of alliances in internet-based supply exchanges. Management Science 2005; 51; 92-105.

Hamlen SS, Hamlen WA Jr, Tschirhart J. The use of the generalized shapley allocation in joint cost allocation. The Accounting Review 1980; 55; 269-287.

Heijboer G. Mathematical and statistical analysis of initial purchasing decisions. Ph.D. thesis, University of Twente: Enschede; 2003.

Hendrick TE. Purchasing consortiums: horizontal alliances among firms buying common goods and services what? who? why? how? Center for Advanced Purchasing Studies, Focus study: Tempe; 1997.

Huber B, Sweeney E, Smyth A. Purchasing consortia and electronic markets: a procurement direction in integrated supply chain management. Electronic Markets 2004; 14; 284-294.

Jeuland AP, Shugan M. Managing channel profits. Marketing Science 1983; 2; 239-272.

Johnnson PF. The pattern of evolution in public sector purchasing consortia. International Journal of Logistics Research and Applications 1999; 2; 57-73.

Keeney RL, Raiffa H. Decisions with multiple objectives. John Wiley: New York; 1976. 
Loehman ET, Winston A. A generalized cost allocation scheme. In: Stevens A, Lin Y, (Eds.), Theory and measurement of economic externalities. Academic Press: New York; 1976.

Moulin H. Axiomatic cost and surplus-sharing. In: Arrow KJ, Sen AK, Suzumura K, (Eds), Handbook of social choice and welfare. North-Holland: Amsterdam; 2001.

Munson C, Rosenblatt M. Theories and realities of quantity discounts: an exploratory study. Production and Operations Management 1998; 7; 352-369.

Nollet J, Beaulieu M. The development of group purchasing: an empirical study in the healthcare sector. Journal of Purchasing and Supply Management 2003; 9; 3-10.

Nollet J, Beaulieu M. Should an organization join a purchasing group? Journal of Supply Chain Management 2005; 10; 11-17.

Rosenblatt MJ, Lee HL. Improving profitability with quantity discounts under fixed demand. IIE Transactions $1985 ; 17 ; 388-395$.

Rozemeijer F. Creating corporate advantage in purchasing. Ph.D. thesis, Technical University of Eindhoven: Eindhoven; 2000.

Schotanus F. Enhancing trust and stability in purchasing consortia: fair allocation of gains. Proceedings of International Purchasing and Supply Education and Research Association conference: Catania; 2004. p. 676-685.

Schotanus F. Cooperative purchasing within the United Nations. Proceedings of International Purchasing and Supply Education and Research Association conference: Archamps; 2005. p. 961-973.

Schotanus F. A basic foundation for unraveling quantity discounts: How to gain more insight into supplier cost mechanisms? Proceedings of International Purchasing and Supply Education and Research Association conference: San Diego; 2006.

Shapley L. A value for n-person games. In: Kuhn H, Tucker A (Eds), Contributions to the theory of games. Princeton University Press: Princeton; 1953.

Sorenson J, Tschirhart J, Whinston A. A theory of pricing under decreasing costs. The American Economic Review 1978; 68; 614-624.

Tella E, Virolainen VM. Motives behind purchasing consortia. International Journal of Production Economics 2005; 93-94; 161-168.

Viswanthan S, Wang Q. Discount pricing decisions in distribution channels with price sensitive demand. European Journal of Operational Research 2003; 571-587.

Zentes J, Swoboda B. Allied groups on the road to complex networks. Technology in Society $2000 ; 22 ; 133-150$. 